\title{
Hospital doctors' Opinions regarding educational Utility, public Sentiment and career Effects of Medical television Dramas: the HOUSE MD study
}

A career in medicine has long been considered an apprenticeship, with mentors providing guidance to their trainees. The word mentor finds its origins in Greek mythology. In Homer's Odyssey, the confidant of king Odysseus, Mentor, was trusted to guide his son and oversee his education while Odysseus fought in the Trojan War. ${ }^{1}$

The modern practice of medicine, with an emphasis on shift work, has made the classical mentor-mentee relationship more challenging, ${ }^{2}$ but the modelling of one's practice on observed social and clinical traits of a mentor or role model remains. ${ }^{3}$ Moreover, such exposures can be factors in students' decisions to pursue a career in medicine and even in their subsequent choice of specialty. ${ }^{4}$

The eventual choice of role model is often a personal one and may not even involve one's own supervising senior, although it is often based on clinical experiences. ${ }^{5}$ While knowledge and clinical competence have been cornerstones of role model selection, growing evidence suggests that factors relating to personality such as compassion, good communication and enthusiasm may in fact have more influence on the expanding minds of trainees. ${ }^{6}$ Further compounding this, in some educational situations, less than half of senior clinicians were subsequently identified as being excellent role models. $^{7}$

While social interactions with parents, teachers or even peers may impact on personality, outlook and practice, other media such as literature and television (TV) have been demonstrated to be significant components of this role model hypothesis. ${ }^{8}$

\section{Abstract}

Objectives: To evaluate the opinions of practicing clinicians on medical television dramas and the effects these series have on society as well as their own practice.

Design, setting and participants: Observational study using a structured questionnaire disseminated among doctors of all grades and specialties at one tertiary centre and two large secondary care district general hospitals in Wales, United Kingdom. Three hundred and seventy-two questionnaires were distributed over a 3-month period, with 200 completed questionnaires received (response rate, 54\%).

Main outcome measures: Frequency and reasons for watching these programs, and opinions regarding realism, educational value and public perception, evaluated by doctors' grades and specialties. Identification of work practice with any observed traits in fictional doctors was also analysed.

Results: $65 \%$ of doctors surveyed admitted to watching these programs on more than one occasion. Junior doctors (interns and resident medical officers) were more regular viewers. Most doctors who admitted to watching medical dramas did so for entertainment purposes (69\%); $8 \%$ watched for educational purposes and, of these, $100 \%$ watched House $M D, 82 \%$ felt that these dramas were unrepresentative of daily practice, and $10 \%$ thought that they accurately portrayed reality. Most of the positive responses were from junior doctors. 61\% of doctors identified some aspect of their clinical practice with another doctor (fictional or non-fictional; most junior doctors identified with a fictional doctor, compared with non-fictional role models for more senior practicing clinicians.

Conclusions: This survey shows that a large body of the medical workforce watches medical television dramas and that such programs exercise a growing influence on the practice of junior doctors, particularly those in physicianly specialties. The reasons for certain role model selections remain unknown and may require further evaluation.

Medical TV programs have grown in popularity from the 1960s onwards and are now considered a staple of primetime TV. ${ }^{9}$ It has only been in more recent years that the effects of these health and illness TV narratives have been studied in greater detail.

Although their true purpose has been one of entertainment, much of their appeal is based on the perception that they are an accurate reflection of reality. ${ }^{10}$

It has been well accepted that TV can have an impact on society, increasing knowledge and influencing behaviour. ${ }^{11}$ TV medical dramas have also been shown to be of educational worth to patients ${ }^{12}$ and even doctors. ${ }^{13}$

However, they have occasionally come under criticism for unrealistic medical content, ranging from demonstration of intubation technique $^{14}$ to cardiopulmonary resuscitation (CPR). ${ }^{15}$ Frequently, in CPR situations on TV compared with actual practice, there is a higher volume of trauma cases as an underlying aetiology. Further, these scenarios often show considerably younger patients than those seen in routine CPR and survival to discharge is much better than clinically encountered. ${ }^{16}$ 
Concerns that this may influence the attitudes of members of the public who watch these dramas for educational purposes remain.

More recently, there has been a growing emphasis on the use of these programs as educational resources. ${ }^{17}$ In particular, some of the established role model personality traits such as ethical astuteness, communication and empathy have been sufficiently demonstrated in these series to warrant use in undergraduate teaching videos. ${ }^{18}$ Although much of the learning that can be gleaned from observing the practices of $\mathrm{TV}$ doctors has focused on perceived softer undergraduate educational domains, ${ }^{19}$ their use in postgraduate settings is also increasing. ${ }^{20}$

TV is a medium through which many health care workers not only take their minds off work, but also reflect both consciously and unconsciously on experiences. Students and doctors do indeed watch these programs at least as often as the general public does and, when questioned, are quite positive regarding them. ${ }^{21}$ Although not yet demonstrated, watching these series may form an early part of any role modelling or identification with certain character traits that both trainee and established medical practitioners may have.

\section{Methods}

A structured questionnaire was distributed among doctors of all grades and specialties in three large teaching hospitals in Wales, United Kingdom (Morriston Hospital, Singleton Hospital and Princess of Wales Hospital) within the Abertawe Bro Morgannwg (ABM) University Health Board, to allow capture of data from a diverse range of specialties. These were disseminated through various different locations, including departmental meetings and on-call rooms.

Questions related to respondents' gender, specialty and grade, whether they watched medical TV dramas and their opinions regarding them, and whether they identified with characters from these programs (and

\begin{tabular}{ll}
1 Grade and specialty of respondents $(n=200)$ & \\
Grade and specialty & No. (\%) \\
\hline Grade & $49(24.5 \%)$ \\
Intern & $60(30.0 \%)$ \\
Core trainee (RMO) & $36(18.0 \%)$ \\
Registrar (specialist trainee) & $55(27.5 \%)$ \\
Consultant & \\
Specialty & $83(41.5 \%)$ \\
Medical & $36(18.0 \%)$ \\
Surgical & $27(13.5 \%)$ \\
Acute non-medical & $20(10.0 \%)$ \\
Non-acute & $34(17.0 \%)$ \\
GP or GP trainee & \\
\hline RMO $=$ resident medical officer. &
\end{tabular}

if so, who) or with a non-fictional doctor encountered during their clinical careers.

Hospital grades were summarised as consultant, specialist trainee (registrar), core trainee (resident medical officer [RMO]), and foundation doctor (intern). For simplification, specialties was separated into medical, surgical, acute (eg, accident and emergency, intensive care unit, etc) and non-acute (eg, pathology, radiology, etc), although note was made of individual subspecialty answers from within these broader categories.

\section{Statistical analysis}

A cumulative odds ordinal logistic regression with proportional odds was run to determine the effect of grade and specialty on the choice and frequency of viewing of medical TV dramas. Statistical significance was set at $P<0.05$. Statistical calculations were performed using SPSS Statistics, version 21.0 (IBM).

\section{Ethics approval}

Ethics approval was granted by the ABM University Health Board Research and Development Joint Scientific Review Committee.

\section{Results}

Three hundred and seventy-two questionnaires were disseminated and 200 completed questionnaires were returned (response rate, 54\%). Forty-six per cent of individuals completing questionnaires were women and $88 \%$ had graduated from a UK medical school. Grades and specialties of respondents are presented in Box 1.

\section{How often do clinicians watch TV medical dramas?}

One hundred and twenty-nine doctors $(65 \%)$ surveyed admitted to watching TV medical dramas on more than one occasion and $14 \%$ considered themselves to be regular viewers; $15 \%$ of respondents felt that watching them as a school student positively influenced their decision to pursue a medical career.

Junior doctors were five times more likely to have watched these programs as medical students compared with more senior doctors (odds ratio [OR], 5.2; 95\% CI, 2.5-10; $P<0.01)$. The ORs for RMOs and specialist trainees were 3.1 and 2.5 , respectively, in relation to consultants $(P<0.05)$. Further, UK graduates were five times more likely to have watched these medical TV dramas as medical students compared with non-UK graduates (OR, 4.8; 95\% CI, 2.4-9.6; $P<0.01)$.

The most commonly watched TV programs were Scrubs (49\%), House MD (35\%) and ER (21\%). Most doctors who admitted to watching medical dramas did so for 


\begin{tabular}{|llll}
\hline \multicolumn{3}{|c}{2 Most popular fictional television doctor role models } \\
Rank & Doctor & Show & Most popular among: \\
\hline 1 & Elliot Reid & Scrubs & Women, junior trainees \\
2 & Perry Cox & Scrubs & Specialist trainees, physicians \\
3 & Leonard McCoy & Star Trek & Consultants, anaesthetists \\
4 & John Carter & ER & Physicians, acute specialties \\
5 & R Quincy & Quincy ME & Consultants, non-acute \\
& & & specialties (pathologists)
\end{tabular}

entertainment purposes (69\%); into media perceptions of medical $19 \%$ watched because there was practice; and $8 \%$ for educational nothing else on TV; $5 \%$ for insight purposes.

3 Dr Evil (Austin Powers film series) was an interesting selection among
some surgical trainees (Getty Images)

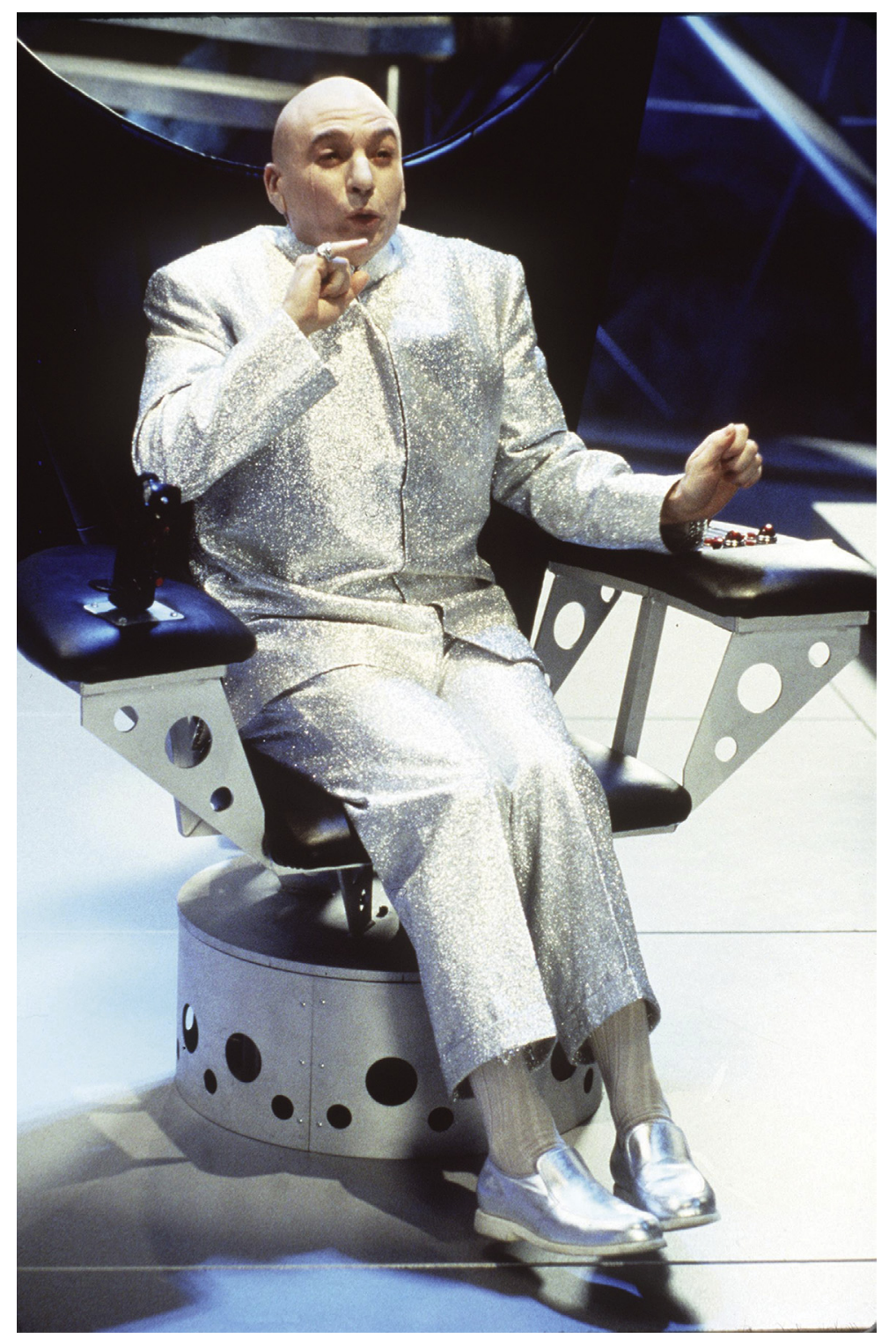

Clinicians' opinions regarding TV medical dramas

We asked individuals if they felt that TV medical dramas were educational, gave doctors a bad name, accurately showed the doctor-nurse relationship, and represented the spectrum of illnesses commonly encountered.

One hundred and three respondents (52\%) felt that these shows displayed no educational value whatsoever, 52 $(26 \%)$ were unsure, and $45(23 \%)$ believed there were some educational benefits from watching them.

Evaluating the spectrum of illness represented in these dramas, $82 \%$ felt that those shown were unrealistic of daily practice. However, 20 respondents $(10 \%)$ thought that they accurately portrayed reality. Most of these positive responses $(16 / 20)$ were from junior doctors. No associations between the belief that medical dramas portrayed realistic life situations and specialty or frequency of viewing were observed.

Grade, specialty and country of qualification had no effect on whether a doctor believed that the programs represented current medical practice. Neither did current frequent watching or having been a regular viewer at undergraduate level.

Twenty-seven per cent of doctors surveyed felt that these programs gave doctors a bad name, although no significant differences were observed between any of the groups.

Only $13 \%$ of respondents felt that medical dramas accurately portrayed the doctor-nurse relationship, most of whom were self-admitted nonregular viewers $(P=0.01)$ and general practitioners or GP trainees (19/25; $P=0.05)$.

\section{Outcomes of watching TV medical dramas}

Thirty per cent of foundation doctors (interns) and $25 \%$ of core trainees (RMOs) felt that watching medical TV programs may have affected their career choice (to any extent) compared with more senior doctors (18\%). 
Compared with consultants, the OR for interns considering that watching medical TV dramas had any effect on their subsequent career choices was 4.8 (95\% CI, 1.6-13.7; $P=0.013)$; for RMOs and specialist trainees, the ORs were 2.5 (95\% CI, 1.3-5.8) and 2.7 (95\% CI, 1.3-5.8) respectively; $P=0.09$ and 0.13$)$.

Specialty and country of qualification did not influence doctors' beliefs that watching medical dramas had an effect on their career choice.

\section{Clinicians' identification with doctors in TV medical dramas?}

A total of 121 respondents (61\%) role modelled aspects of their practice on another doctor (fictional and nonfictional).

Junior doctors, particularly interns and RMOs were more likely to find commonality in their practice with fictional TV characters compared with more senior doctors (OR, 2.7; 95\% CI, 1.3-5.8; $P=0.008)$.

Consultants were most likely not to specify any role models and, if they did so, were more likely to identify themselves with non-fictional characters $(32 / 55)$ compared with other doctors, particularly interns (4/49).

Medical doctors were more likely to identify themselves with a fictional TV character (OR, 3.2; 95\% CI, 1.08-9.43; $\quad P=0.035)$. This was followed by $19 \%$ of acute specialty doctors and $14 \%$ of surgical specialty doctors. Non-acute specialty doctors were least likely to identify themselves with a fictional TV doctor.

The top five most popular fictional role models are shown in Box 2. Leonard McCoy (Star Trek) and Quincy (Quincy ME) were the most popular choices among consultants; the majority of positive responders were anaesthetists and pathologists. A more varied response was seen among physicians and surgeons, but note was made of a peculiar popular choice: Dr Evil (from the Austin Powers film series, Box 3) was named by four trainees, all surgical (three orthopaedic and one general surgery).

\section{Discussion}

There is a known association between clinical role models in undergraduate medicine and career choice. $^{22}$ Therefore, TV medical dramas could potentially influence doctors' and students' opinions and have been found to be a source of entertainment for both health care professionals as well as the wider public. $^{23}$

Fictional doctors have evolved into television heroes and much of their appeal is their on-screen personality

as well as, in some cases, their absolute prioritisation of scientific challenge over social relationships. ${ }^{24}$ Further, much of their appeal is their ability to navigate through difficult ethical dilemmas, to make decisions that are often perceived by clinical trainees as being positive ones. $^{25}$

Although clinicians watching these programs appear to do so predominantly for entertainment purposes, we found that those who watch for educational reasons show that junior trainees exposed to this genre of TV

4 House $M D$ was considered the most educational among respondents (Getty Images)

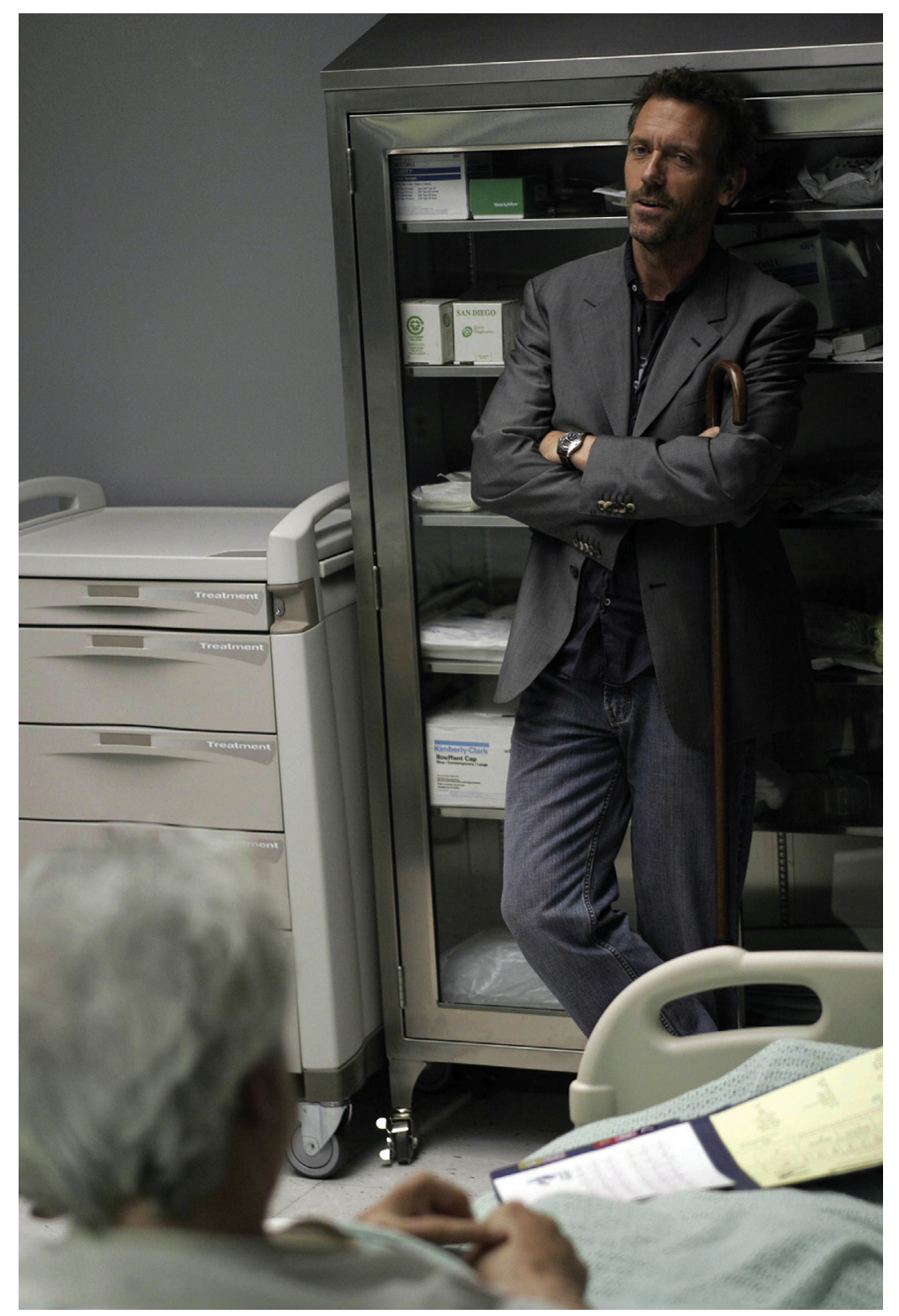


entertainment are more influenced by these series than their more senior counterparts. Interestingly, all respondents who admitted to watching TV medical dramas for educational reasons watched House $M D$ (Box 4), perhaps suggesting that they value its learning input.

In keeping with previous studies, ${ }^{14-16}$ most doctors felt that a large proportion of what was televised may not be a true representation of clinical practice; however, suggestions that more junior trainees believe this to be so could be explained by their relative lack of clinical experiences to date.

Identifying aspects of one's practice with witnessed exposures has been a cornerstone of the role modelling theory, but data generated from this questionnaire-based study suggest some interesting differences between specialties. Doctors who answered negatively to currently viewing or having ever viewed this type of program were least likely to admit to having been influenced into a career in medicine on the basis of TV medical dramas, thus validating the data.

It is to be assumed that consultants may look on their past seniors as role models to identify commonality of practice but the high proportion of respondents among all grades who admitted to being influenced, at least in part, by medical TV dramas suggests a much higher effect than anticipated.

Further, differences between specialities - for example, medical doctors identifying more with TV doctors compared with their surgical peers - might be explained by the sizeable volume of medically themed programs as opposed to more surgical ones. It is plausible, however, that some of the core learning traits seen in physicianly specialties, particularly regarding difficult diagnostics and ethical dilemmas, strike a chord with this group of clinicians. Specific choice of TV doctor hero as a potential role model will require further evaluation. Motivations for the popular choice of a Star Trek character among anaesthetists may include an interest in futuristic technology. Likewise, the interesting preference for Dr Evil among some surgical trainees may be due to an interest in world and/or career domination, or it may be suggestive of professional ambition rather than a display of true megalomaniac traits.

While we may be some years from continuing medical education creditation obtained from Saturday evening viewing, this study does suggest that the current generation of junior doctors relies on medical TV dramas for entertainment and education in parallel. Further observation may show some interesting effects during career progression, particularly regarding the atypical answers we received to our questions about TV doctor identification.

Competing interests: No relevant disclosures.

C 2015 AMPCo Pty Ltd. Produced with Elsevier B.V. All rights reserved.

References are available online at www.mia.com.au. 
1 Steiner JF, Curtis P, Lanphear BP. Assessing the role of influential mentors in the research development of primary care fellows. Acad Med 2004; 79: 865-872.

2 Blencowe NS, Parsons BA, Hollowood AD. Effects of changing work patterns on general surgical training over the last decade. Postgrad Med J 2011; 87: 795-799.

3 Monkhouse S. Learning in the surgical workplace: necessity not luxury. Clin Teach 2010; 7: 167-170.

4 Goldacre MJ, Goldacre R, Lambert TW. Doctors who considered but did not pursue specific clinical specialties as careers: questionnaire surveys. $J R$ Soc Med 2012; 105: 166-176.

5 Kravet SJ, Christmas CM, Durso S, et al. The intersection between clinical excellence and role modeling in medicine. J Grad Med Educ 2011; 3: 465-468.

6 Paice E, Heard S, Moss F. How important are role models in making good doctors. BMJ 2002; 325: 707-710.

7 Wright SM, Kern DE, Kolodner K, et al. Attributes of excellent attendingphysician role models. $N$ Engl J Med 1998; 339: 1986-1993.

8 Corradi RB, Wasman M, Gold FS. Teaching about transference: a videotape introduction. Am J Psychother 1980; 34: 564-571.

9 Davin S. Healthy viewing: the reception of medical narratives. Sociol Health Illn 2003; 25: 662-679.
10 O'Connor MM. The role of the television drama ER in medical student life: Entertainment or socialization? JAMA 1998; 280: 854-855.

11 Howe A., Owen-Smith V, Richardson J. The impact of a television soap opera on the NHS cervical screening programme in the north west of England. J Public Health Med 2002; 24: 299-304.

12 Kennedy MG, O'Leary A, Beck V, et al. Increases in calls to the CDC national STD and AIDS hotline following AIDS-related episodes in a soap opera. J Commun 2004; 44: 287-301.

13 Dahms K, Sharkova Y, Heitland P, et al. Cobalt intoxication diagnosed with the help of Dr House. Lancet 2014: 383: 574.

14 Brindley PG, Needham C. Positioning prior to endotracheal intubation on a television medical drama: perhaps life mimics art. Resuscitation 2009; 80: 604.

15 Harris D, Willoughby $H$. Resuscitation on television: realistic or ridiculous? A quantitative observational analysis of the portrayal of cardiopulmonary resuscitation in television medical drama. Resuscitation 2009; 80: 1275-1279.

16 Gordon PN, Williamson S, Lawler PG. As seen on TV: observational study of cardiopulmonary resuscitation in British television medical dramas. BMJ 1998; 317: 780-783.
17 Reid G. The television dramadocumentary (dramadoc) as a form of science communication. Public Underst Sci 2012; 21: 984-1001.

18 Hirt C, Wong K, Erichsen S, White JS. Medical dramas on television: a brief guide for educators. Med Teach 2013; 35: 237-242.

19 Spike J. Television viewing and ethical reasoning: why watching Scrubs does a better job than most bioethics classes. Am J Bioeth 2008; 8: 11-13.

20 Johnson JM, Beresin EV, Stern TA. Using Breaking Bad to teach about defense mechanisms. Acad Psychiatry 2014; 38: 716-719.

21 Chory-Assad RM, Tamborini R. Television exposure and the public's perception of physicians. J Broadcast Electron Media 2003; 47: 197-215.

22 Wright S, Wong A, Newill C. The impact of role models on medical students. J Gen Intern Med 1997; 12: 53-56.

23 Lee TK, Taylor LD. The motives for and consequences of viewing television medical dramas. Health Commun 2014; 29: 13-22.

24 Strauman EC, Goodier BC. The doctor(s) in house: an analysis of the evolution of the television doctor-hero. J Med Humanit 2011; 32: 31-46.

25 Weaver R, Wilson I. Australian medical students' perceptions of professionalism and ethics in medical television programs. BMC Med Educ 2011; 11: 50. 